Proceedings of the 2004 IEEE

T1B2

\title{
NoSEBrEaK - Attacking Honeynets
}

\author{
Maximillian Dornseif Thorsten Holz Christian N. Klein
}

\begin{abstract}
It is usually assumed that Honeynets are hard to detect and that attempts to detect or disable them can be unconditionally monitored. We scrutinize this assumption and demonstrate a method how a host in a honeynet can be completely controlled by an attacker without any substantial logging taking place.
\end{abstract}

\section{INTRODUCTION}

At the Laboratory for Dependable Distributed Systems , at RWTH Aachen University, Germany, we run a Linux based honeynet for gathering information on security incidents. The scientific method dictates that we must attack ' our own assumptions vigorously to get meaningful results. ' Under the code name "NoSEBrEaK" we attacked our orig' inal assumptions about undetectability and monitorability ' of honeynets by turning the table and taking the view of an attacker trying to control a honeypot. In the following ' paper we present the results of this red team approach.

Honeynet researchers want to "learn the tools, tactics, ' and motives of the blackhat community and share the lessons learned" [1. Therefore honeynets must provide a ' way to capture the activities of an intruder in order to provide some information about his actions and the tools he ' used. The traditional method used to capture keystrokes and other activities on Unix Systems is to patch /bin/sh 'the standard interpreter - in order to log every keystroke of an intruder to a special file. Other possibilities are to redi'rect the output of syslogd to another host on the network or to record and analyze all network traffic with tcpdump and other tools. But network monitoring attempts are doomed to fail if the intruder utilizes encryption - for example by using SSH or SSL to connect to the honeynet - to protect his communication channel. Then all captured data is useless because the information can not be decrypted without the appropriate key.

Trojaned binaries on the honeynet that record all keystrokes by the attacker can be circumvented if the attacker installs his own binaries, which is a common way nowadays. Thus, a new way to capture the activities of an intruder on an Linux system was developed: A kernelbased rootkit called Sebek [2] is able to record all data

dornseif@informatik.rwth-aachen.de - Laboratory for Dependable Distributed Systems, RWTH Aachen University.

holz@i4.informatik.rwth-aachen.de - Laboratory for Dependable Distributed Systems, RWTH Aachen University.

kleinc@cs.bonn.edu - University of Bonn. accessed by users via the read( $)$ system call on the honeynet. It replaces the normal read() system call with a new entry in the system call table pointing to its own version of this system call. It is then able to record all data accessed via read () 3. Because Sebek lives entirely in kernel-space and has access to all data read, this rootkit is able to access most communication unencrypted and it can for example log SSH-sessions, recover files copied with SCP and record all passwords used by intruders. The recorded data is sent via UDP to the Sebek server, the other part of Sebek's client/server architecture. This transmission is done by modifying the kernel in order to hide these packets such that an intruder can not see them. In addition, all network counters and data structures have to be readapted in order to make detecting these changes more difficult. Further information about Sebek and its architecture can be found in 3 .

In this paper we show that a qualified attacker - in contrast to an unsophisticated attacker or autonomous malware like worms - has several ways to detect and circumvent Sebek, the primary data capture tool used by honeynet researchers to capture the attackers' activities on a honeynet.

The paper is outlined as follows: Section 1 gives an overview of related work in the field of detection of honeynets. Several ways to detect, disable and bypass Sebek, mainly implemented in our proof of concept toolkit Kebes, are presented in section III] Directions of further work are outlined in section [IV and we conclude this paper with section $\nabla$

\section{RELATED WORK}

Unfortunately, there is little scientific literature on the topic of honeynets and none on detecting or circumventing data capture mechanisms in honeynets. The only related work was published in two fake issues of Phrack [4], a Hacker magazine that is famous for its articles in the blackhat community.

In Phrack 62 [5] an article entitled "Local Honeypot Identification" was published. It describes a method to disable Sebek by just overwriting Sebek's read() system call in the system call table with the original value and thus disabling Sebek. We could not verify that this is easily achievable since it seems that the position of the original system call can not easily obtained. Other techniques to 
detect the existence of Sebek on a host were also presented in the article. They include detection of an outgoing connection limit used on most honeynets and the existence of snort_inline [6], an intrusion prevention systems that modifies packets that look harmful.

Another idea to defeat Sebek was published in issue 63 of the Phrack magazine [7: The article "Advanced Honey Pot Identification" describes a way to search through memory and look for characteristic symbols used by Sebek. The accompanying code is claimed to be able to reconstruct several highly sensitive bits of Sebek data, including the magic number and port numbers used by Sebek to identify and hide its communication from others. After publication of the article, multiple changes in Sebek's code were released to counter this identification techniques, including randomization of the symbol names used.

Issue 61 of the Phrack magazine contained an article on detection of hidden kernel modules in its "Linenoise" section [8]. The article describes a brute force method for detecting hidden modules by looking for what appears to be the key module structure. In our tests this method was able to locate a hidden Sebek module.

There also is a tool named chkrootkit which "is a tool to locally check for signs of a rootkit" 9]. While the documentation claims that the tool is able to detect Sebek, this is only true for very old versions of Sebek. Detecting is accomplished by searching/proc/ksyms for the string "sebek".

\section{SEVERAL WAYS TO DETECT AND CIRCUMVENT SEBEK}

In this section we present several ideas and ways to detect and circumvent Sebek in order to prevent logging of user activities and thus effectively preventing honeynet researches to collect information about intruders.

We first present ideas to detect Sebek, followed by sections dealing with disabling of Sebek and detection of the honeywall. In the last section we describe several ideas and ways to circumvent Sebek, in particular our implementation Kebes, a tool to effectively bypass Sebek.

\section{A. Detecting Sebek}

Sebek is a kernel module, so it leaves several traces of its existence in memory. We now describe ways to track down those traces, thus leading to the detection of Sebek.

One of our first experiments originally was aimed at making logging by Sebek unreliable. By accident we found an easy way to detect Sebek which can be used even by unprivileged local users:

user@honey: dd if=/dev/zero of=/dev/null bs=1

This simple command results in backward running packet and byte counters of the network interface, so even an unprivileged user was able to detect Sebek by checking the output of ifconfig. After we pointed this out to
Sebek's maintainers, this issue was mitigated with version 2.1.7 of Sebek.

To understand why using dd should lead to unreliable logging by Sebek, one has to take a closer look at the logging process: As explained above, Sebek replaces the normal sys_read() call in the system call table with its own version and is thus able to record all data accessed via read (). The logging data is then transfered in a covert way via UDP over the network 3 . Therefore, if we are reading a single byte via a read(1), Sebek has to transfer nearly 100 Bytes of data including all the network headers over the network to the host doing the logging. So if we are able to do a read(1) some tens of thousands of times per second, this will lead to a congested network and finally to dropped packets. And we are able to identify a congested network with the help of the ping command.

This leads to another way to detect Sebek, which we call the $d d$-attack, since it can accomplished with a simple command like dd, as shown above. To detect Sebek on a host, we first ping a local IP-address (e.g. the gateway) in order to get an overview of the current performance of the network. Then $d d$ is started in the background and we rerun the ping command. If Sebek is installed on a host, this leads to an enormous increase in the average round-trip time. In practical tests of this attack the average round-trip time grew from 0.7 milliseconds to over 4800 milliseconds.

But the fix in Sebek which was introduced in version 2.1.7 leads to a new way to detect Sebek: In an attempt to control the backward moving transmit-counters, the counters are untouched and Sebek modifies the output routines for / proc/net/dev to deduct the number of Sebek packages from the total number of transmitted packages just before output. This enables us to compare dev->get_stats ()->tx_bytes with the values listed in /proc/net/dev for every ethernet device on the system. Differences can only be explained by the presence of Sebek.

Another way to detect Sebek is similar to the approach published in Phrack 63 [7: Sebek is a kernel module and it has to use some kernel memory in order to be able to store its variables like magic number and source or destination port. Version 2.1.7 uses some randomization and other stealth techniques in order to prevent easy ways to find these variables, but Sebek leaves some traces in memory that can be found:

- Since Sebek by default chooses a random number below 1000000000 as the module name it is easy to create heuristics to detect the Sebek module purely by its name in the lists of modules generated by the methods outlined in [8]. A small obstacle in doing so is that there are legitimate modules with purely numeric names like 8390, a driver for a generic Ethernet chipset.

- Helpful in the detection of Sebek symbols is the knowledge that all it's symbol names start with a letter, followed by a number with up to three digits. 
- Also helpful is the fact that a conversion table to translate IP addresses into integers is left in memory. In addition to falsify /proc/net/dev output, Sebek has to keep more than 200 bytes of strings in memory, which can be located without great effort.

- The hidden Sebek module still has a complete module header structure in memory. This structure can be found with very little effort 8 and used to locate Sebek's code (e.g.its cleanup function) and variable storage.

- While the placement of variables in Sebek's variable storage block is different every time Sebek is compiled, it is still possible to locate the variable storage block via the module header. That information can be used to successfully guess most variables like magic number, source and destination port with a few tries.

All these traces can be found by traversing memory and looking for appropriate structures and thus leading to a way to detect hidden kernel modules, identify the Sebek kernel module and extract secrets from the module. We are able to implement this approach and it leads to another way by which Sebek can be detected on a host.

In [5], a way to detect the changes in the system call table for Solaris is proposed. We carried this idea forward and implemented it: When Sebek installs its read() monitoring function, it replaces the function pointer in the system call table at NR_read with a pointer to its own function, declared in the module. While on a system without Sebek, the system call pointers are close to one another in memory, this changes if Sebek is loaded into memory. The address of Sebek's function does not lie near the address of sys_write, the successor of sys_read in the system call table. Thus, if the addresses of sys_read and sys_write lie far apart (e.g. more than 4096 byte), we conclude that the system call table was modified by some rootkit. Furthermore, there is another similar way to detect Sebek: After Sebek has replaced the function pointer in the system call table, sys_read points inside the memory range of a module. This is a clear sign that the system calls were changed and a rootkit is present. We wrote a program that searches through memory and finds both of these anomalies.

As an example, Figure 1 shows the places of two system calls in memory before and after Sebek is loaded. One can clearly see that the system call table was modified and something malicious is happening.

\section{B. Disabling Sebek}

After having shown several ways to detect Sebek, we now point out sundry possibilities to disable it. One evident possibility to disable Sebek is simply rebooting. Currently Sebek offers no possibility to be loaded automatically on system startup, but this can easily be achieved by customizing the shell scripts. But integrating the installation of Sebek into the boot process would result in leaving a lot of evidence of Sebek's existence and an avenue for removing

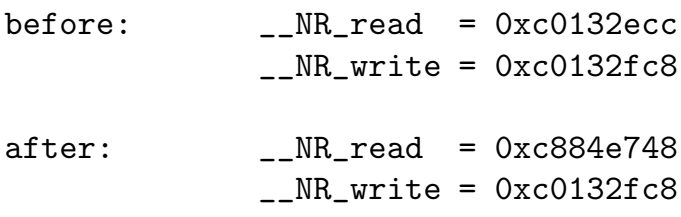

Fig. 1

AdDresses of two System CALls Before AND AFter SEBEK IS LOADED.

this boot scripts and rebooting to disable Sebek.

Another possibility is more complicated: Reconstruction of the original system call table and thus effectively disabling Sebek. We were not able to reproduce results from [5]: Since sys_read is not an exported kernel symbol, we can not simply obtain the address of the original sys_read. It might be feasible to find the memory address of the original sys_read call through parsing the kernel image on disk. An alternative would be searching through the memory of the Sebek kernel module to obtain the address of the original system call.

Probably the most elegant solution is asking Sebek to remove itself. If we are able to obtain the address of Sebek's cleanup () function we can call this function which will result in Sebek restoring the original state of the system call table and thus disabling further logging. Since we have already shown that the key module structures can be reconstructed, we are able to implement this approach to disable Sebek.

\section{Detecting the Honeywall}

Most honeynet operators rightfully fear liability if intruders use the honeynet as a stepping stone to attack systems of third parties. Therefore, two main techniques are deployed to minimize the risk of a successful attack on third parties' systems: First, outgoing traffic is heavily restricted to values like only 15 outgoing TCP connections per day. Such extreme means of traffic shaping are trivial to detect: We only have to initiate a large number of outgoing connections (SYN packets to other hosts) and watch if after some number of connections we get blocked by the honeywall.

The second means of avoiding outbound attacks by honeynets is the usage of a piece of software named snort_inline 6]. This software acts as an inline packet modification engine rewriting what looks like something considered dangerous to something harmless. The example for this technique used by the honeynet project all the time is the replacement of the string / bin/sh in shellcode with the string /ben/sh [?]. Another example is the replacement of some characteristic patterns in network traffic in order to render attacks useless, as shown in Figure 2

This allows us to detect a honeywall trivially by constructing some network communication containing strings 


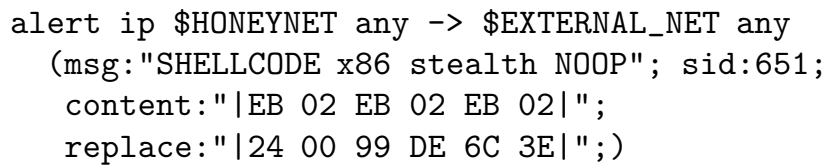

Fig. 2

SNORT_INLINE-RULE TO MODIFY PACKETS WITH X86-SHELLCODE.

which match snort_inline's rewriting database and check if the communication is received in unaltered form.

We are able to implement both ways to detect the honeywall and our experiments show that a honewall can be easily detected this way.

\section{Circumventing Logging on a Honeynet}

\section{D.1 Overview}

Logging on a honeynet can be circumvented by making logged data unintelligible, making logging itself unreliable or using ways not to be logged at all.

There is little we can do about the logging of all network communications done by the honeywall. We might be able to penetrate the honeywall and wipe data from the machine, but we cannot rely on this option. This means that we have to use cryptography in network communications whenever possible to avoid logging. The main problem in doing so is how to deliver our initial exploit to the target in an encrypted fashion. In many instances we will not be able to deploy encryption when transmitting the initial exploit. Notable exceptions are exploits delivered via HTTPS: If the target offers the HTTPS service we have a wide range of opportunities to exploit the webserver, vulnerable CGI-Programs or other programs accessible via the web interface while being able to communicate in encrypted form and therefore unintelligible for network traffic logging.

To circumventing logging by Sebek we again take a look at how Sebek obtains this data: Sebek has all data available which is gained by the sys_read kernel function. The PID and UID of the process calling read() is recorded, as well the number of the filedescriptor being read, the first 12 characters of the filename of the program calling read() and the data being read itself. Nearly as interesting as what is logged is the observation what is not logged. For example, neither is the path of the file being accessed logged nor the path of the program calling read(). This means the adversary doing forensic analysis is restricted to guesswork on what is read by whom. This allows us to clutter up Sebek logging with arbitrary data. For example, we can create a file looking like a legitimate shadow-password file in /tmp and read it via cat. The adversary will probably at first glance assume we were able to read the real shadow-file. We can also copy binaries, which we are going to use, to random names and in doing so give the adver- sary a hard time guessing which programs were executed. While we have no direct control over the PID, we can add frequent executions of fork () coupled with termination of the parent to keep the PID being a moving target. If such deceptive measures are executed in an automated yet well planned fashion, we can mix the commands we issue to directly accomplish our objectives with thousands of dummy commands. Hence, we can generate so much logging data that forensic analysis will be made much more complicated.

Another option is to make logging by Sebek unreliable. As shown in section $\amalg$ II-A this can be archived via the $d d$ attack, in which dropping of Sebek packages should take place. The percentage of packages dropped by the $d d$ attack varies greatly. So while this method of circumventing is not fully reliable, it can be expected that the adversary will not be able to fully reconstruct a session while a dd-attack takes place.

The preferred method to circumvent logging is avoiding it at all. In order to avoid recording of the activities on a honeynet which is using Sebek, we have to avoid using read () system calls, since all data which is read via read () system calls is transfered over the network to a logging host. One way of avoiding $\operatorname{read}()$ is to use mmap() to map files directly into memory and therefore bypassing logging. This allows us to read all regular files on the system without Sebek noticing this at all. Unfortunately, device files and files on special filesystems like /proc can not be read in this manner.

For reading data from the network we can exchange the read () system call for recv() which will not be logged by Sebek. Unfortunately, this is of little practical value since we have to assume that all network traffic is captured by the adversary so we have to encrypt network traffic. So while using recv() provides only limited help to keep data secure at least it hides the fact from the adversary which process is participating in the network communication.

It is notable that executing programs via exec() does not involve read(). This basically means that we can in theory execute arbitrary programs without logging by Sebek taking place. It turns out that this is only true to a limited extent: At program startup, the dynamic linker loads various dynamic libraries into memory. This is done mostly via mmap () but also sometimes via read(). So if we execute a program, there is a chance that Sebek is logging a read()-call of a library by that program.

\section{D.2 Kebes: A circumvention device}

To experiment with some of the limitations in Sebek, we constructed a proof-of-concept toolkit called Kebes. Kebes is written in the high-level programming language Python and is designed to allow a wide variety of actions being taken on the target system without read() being called.

While Kebes uses a layered approach in communication and is therefore not restricted in the type of communica- 
tion it uses, we opted for simplicity and decided to use a traditional client/server setup: The server resides on the target, listening on a TCP socket and forking childs for handling connecting clients.

The TCP connection is used by a layer that we call the crypt layer, which provides an AES-encrypted messagebased communication channel for Kebes. Besides the encryption itself, the crypt layer provides some support services like random padding and compression via $z l i b$ where we use a random compression level between 0 and 9 to further obfuscate the message length. The encryption is set up by utilizing a Diffie-Hellman key exchange, so there are no pre-shared secrets between the Kebes server and client. Using plain Diffie-Hellman makes the crypt layer vulnerable to man-in-the-middle attacks. But our concern constructing Kebes was not to defeat the active attacker; such a scheme must ultimately fail since the active attacker probably has also complete control of the target host we use to run the Kebes server. Rather our goal was to make ex-post analysis of network, filesystem and Sebek data hard if not impossible.

Above the crypt layer we use the so called Kebes layer. The Kebes layer consists of serialized Python data structures: Each command message consists of a list of commands whereas each command consists of a tag considered by the server as an opaque identifier of the instance of command invocation designed to allow asynchronous return of replies. The second element is a string identifying the command to be executed and the third element is a list of parameters, which can consist of command specific Python objects.

Tapping in the strengths of the highly dynamic Python language, the Kebes server initially understands just a single command: ADDCOMMAND. All further commands desired by the client are dynamically loaded via the network in the server process. This basically means that the server is only a communication stub which is dynamically extended via an encrypted network channel. This does not only make maintenance much easier, since updates can be pushed by the client into the server without restarting the server, but also should make forensic analysis of Kebes' inner workings much harder: As long as no paging or core dump of the Kebes process occurs, there should be no permanent record of the more advanced functionality added to the server by pushing the code for that functionality to the client.

The Kebes commands implemented this way include besides trivial to implement commands for listing directories, getting file information, creating files and getting system information some more complex commands: Deleting files is implemented in the most secure manner possible in a portable way in a high level language. We rename the file to a random name, overwrite its content by a slightly longer random pattern, sync the file to disk and repeat the whole procedure various times before finally unlinking the file.

Reading a file is implemented by using mmap () to instruct the virtual memory system to map the file contents into the address space of the Kebes server. A copy of this memory area is then simply sent over the network to the Kebes clients. As shown above, this method of reading files is not logged by Sebek.

Files are executed by first redirecting file descriptors for stdout and stderr to write to files and creating a file from which stdin will read. While using a file for stdin creates a risk of being recovered - even if we try secure deletion as outlined above - we see little additional risk of compromising extra information, since data read by the process we are executing can be logged by Sebek anyway. Redirecting output to a file is the only way to allow us using mmap() to read the output without calling read(), although we gain this advantage by the increased risk that secure deletion of this files is unsuccessful and forensic diskanalysis will reveal the output saved in the files. After the files are set up, the Sebek server forks, uses $\operatorname{exec}()$ to execute the desired commands and after termination of the child process returns process id, status, and output to the Kebes client.

We also created an extended version of the execution command which is able to receive a binary from the client, saves it under an arbitrary name, executes it and finally securely deletes the binary again. This version of the execution command can also create a temporary copy of an existing command on the target host with an arbitrary name, executes the copy and then deletes it. While we have only limited control on where the programs executed by us use read (), we make analysis of data collected by Sebek much harder by controlling the process names under which the read activity is logged.

For highly sensitive code, which should fail under no circumstances in the hands of the adversary, we prototyped a way to execute code without ever writing it to disk. To support this, the Kebes server can compile an extension module based on C-Code for Python called shellcode-executer on the fly. The shellcode-executer extension takes a Python string - which can contain 0-bytes - and lets the processor directly jump into that string allowing the execution of arbitrary machine code by Kebes. This idea could be extended until Kebes could execute complete ELF binaries received by the network as pioneered in [10].

The main challenge when implementing Kebes was to get the entropy for the secret in the initial Diffie-Hellman key exchange: Since /dev/random can not be read by mmap(), we would have to resort to read(), which would allow an adversary to gain knowledge of the entropy used to set up the Diffie-Hellman key exchange and thus reconstruct the communication keys. We first contemplated to fork() another process, which would perform an dd-attack by pushing as much data as possible through $\operatorname{read}()$. This would 
be done by reading /dev/zero hoping that this would result in an overload of the network carrying the data captured by Sebek and thus result in not all data processed by read() being logged. While it turned out that this generally works as expected, it is extremely unstealthy. We now use clock jitter, thread races and the contents of some files to get the entropy from.

We start two threads doing exactly the same: for every file we find in the /var and /tmp filesystem of the host, we add to a temporary list:

- A high resolution timestamp when processing started.

- The ID of the thread to encode the hard to predict fact which thread is processing a file first.

- SHA-1 of the file in question if the file is readable and smaller than one megabyte; if the file is readable but bigger, the last megabyte of the file. Reading is done via mmap () to avoid logging by Sebek.

- A high resolution timestamp when processing stopped.

Every item from this temporary list is then stirred together with it's list position and another timestamp in the 1960 Bit entropy pool. While this method is probably unsuitable for strong cryptography, it seems good enough to seriously slow down forensic examination of network traffic.

\section{D.3 Testing the NoSEBrEaK Ideas}

To test the effectiveness in defeating Sebek by our NoSEBrEaK ideas, we prepared a Linux-honeynet running Sebek 2.1.7. This machine contains a CGI-Program which we prepared to contain a stack-overflow. We also planted a SUID-root executable with an stack-overflow in parameter processing. Instead of this vulnerabilities introduced by ourselves, we could have exploited known vulnerabilities in the Linux installation on the honeynet, but for testing purposes we wanted to be somewhat independent of distribution flavors and software versions.

We prepared an exploit for the CGI-Program utilizing shellcode that is able to load a binary from the network and execute it. As the binary to be transferred, we created a compound binary consisting of the Python runtime environment the byte code compiled Kebes server. We also created an exploit for the vulnerable SUID-root tool, which is able to execute arbitrary programs with exec (). Finally, we created a Python program packaged again as an compound binary which accesses kernel internal data structures to find the Sebek module, guesses the values used for network addresses, ports and magic value in the Sebek module and the addresses of the module's init() and cleanup () functions. This program is accompanied by a minimal kernel-module which just enables the user to jump to arbitrarily locations in the kernel. By calling this module with the address of Sebek's cleanup () function we can force Sebek to disable itself.

Equipped with this toolset, we first connect via HTTPS to the webserver on our target. Using HTTPS practically renders logging of network traffic ineffective - although analysis of traffic patterns and source address is still possible. We deliver the shellcode via a stack-overflow in the path and the compound binary in the request body. The binary is read by the shellcode, written to disk and executed. This starts the Kebes server which binds to a TCP ports and listens to incoming connections while running under user ID www-data, like the webserver it was spawned off is doing. We connect with our Kebes client to the Kebes server and add dynamically the needed commands to the server. Then we write the binaries used to deactivate Sebek to disk and start the vulnerable SUID-root program with parameters exploiting the vulnerability and starting the Sebek detection and removal program as root. Finally, we again exploit the SUID-root binary, this time to start a Kebes server with root permissions.

After this few relatively easy steps we earned full control over the honeynet machine while none of our activities were logged by Sebek and the recording of network traffic only left the adversary with some encrypted traffic and the cleartext Diffie-Hellman key exchange.

Therefore our experiment rebuts our original assumption that even if an attacker manages to disable Sebek we would at least gain a transcript of the methods used to disable Sebek. We can not trust any data that is logged on a Sebekbased honeynet if there has some encrypted communication between the honeynet an a possible attacker taken place.

\section{FURTHER WORK}

There is a broad range of possibilities to enhance our work in further attacking Sebek. But also several approaches in hardening Sebek against attacks come to mind.

For further attacking Sebek we see lots of possibilities. Some of them include:

- Sebek leaves many traces in memory: It is a kernel module and thus uses some special data structures, it uses characteristic variables and builds UDP packets in memory. Therefore it should be possible to search through memory and detect its existence in several other places than by looking for module structures.

- Disable Sebek by patching the Sebek kernel module at runtime to skip the call to the logging function.

- Burneye, an executable encryption program which is suited to protect ELF binaries on the Intel x86 Linux operating system [1], is very popular among blackhats. With the help of Sebek, the password used by an intruder to decrypt the binary can easily be observed [3]. The implementation of a challenge-response technique for burneye in order to protect the binary would make the decryption much harder.

- Hook into the low level networking layer at the level of the network drivers to unhide Sebek's logging packages.

If we turn the table again and take the view of a honeynet researcher, we see some topics of interest in order to harden 
Sebek and its general approach to log activities of intruders: - Obviously, transforming Sebek to be a kernel patch instead of a loadable kernel module should be aspired. Detecting and removing Sebek if it is not dynamically loaded as a kernel module would be much more difficult and installation after a reboot would become a non-issue. On the other hand, deployment of Sebek would get complicated by doing so. In our opinion this is actually desirable, since honeynets are dangerous and ethically problematic tools and entities wishing to deploy honeypots should be willing and able to got to the pains of patching and compiling a kernel.

- Adding further cleaning of module structures in memory after installing the Sebek kernel module, using a less predictable way of generating the "random" symbol and module names.

- Further obfuscating the placement of Sebek's variables in memory and adding decoy variables.

- Deploying polymorphic techniques to further obfuscate Sebek in memory.

- Using source and destination ports and MAC addressees in addition to the magic number when identifying Sebek packages to extend search space from $2^{48}$ to $2^{160}$ Packets when brute-forcing.

\section{COnClusions}

We have shown that Sebek can be detected, disabled and it's logging circumvented. This knowledge lets us take a completely different view on data obtained by honeynets and on data not obtained by honeynets. While unsophisticated attackers might not be able to circumvent honeynets or not even try to do so, we assume that sophisticated attackers can detect honeynets and disable logging on them if this fits their objectives. If there are already very advanced techniques of detecting and disabling honeynets discussed in the open wild 5, 7, we have to assume that there are much more evolved techniques available to highly advanced attackers. This underlines our apprehension that honeynet technology is only able to gather information of the common crowd of unsophisticated attackers, but has a very small probability of gathering significant information on advanced attackers which would be of much more value to researchers.

\section{ACKNOWLEDGEMENTS}

We owe thanks to Felix Gärtner for making our research possible and Phillip Maihöfer for providing us with test platforms.

Thorsten Holz was supported by the Deutsche Forschungsgemeinschaft (DFG) as a research student in the DFG-Graduiertenkolleg "Software für mobile Kommunikationssysteme" at RWTH Aachen University.

\section{REFERENCES}

[1] "The honeynet project." Internet: http://www.honeynet.org/
[2] "Sebek." Internet: http://honeynet.org/papers/honeynet/tools/sebek/ 2004.

[3] The Honeynet Project, "Know your Enemy: Sebek," November 2003. http://www.honeynet.org/papers/sebek.pdf

4] "Phrack magazine." Internet: http://www.phrack.org/

[5] J. Corey, "Local Honeypot Identification," September 2003. http://www.phrack.org/fakes/p62/p62-0x07.txt

[6] "Snort_inline." Internet: http://snort-inline.sourceforge.net/ 2004 .

[7] J. Corey, "Advanced Honey Pot Identification," Januar 2004. http://www.phrack.org/fakes/p63/p63-0x09.txt

[8] madsys, "Finding hidden kernel modules (the extrem way)," 2003. http://www.phrack.org/phrack/61/p61-0x03_Linenoise.txt

[9] "chkrootkit homepage." Internet: http://www. chkrootkit.org/

[10] m1lt0n, "Keeping Oday Safe," Januar 2004. http://www.phrack.org/fakes/p63/p63-0x08.txt

[11] "Burneye - teso elf encryption engine." Internet: http://www.team-teso.net/releases/burneye-1.0.1-src.tar.bz2 2004. 